\title{
Kräuterbücher (2)
}

Publikationskonflikte in der Renaissance: Leonhart Fuchs und Conrad Gessner im Streit um ihre Pflanzenwerke

\section{Barbara Brauckmann}

Im umfangreichen Nachlass des Pharmazeuten Prof. Dr. Carl Hartwich (1851-1917; siehe auch Teil 1 in Ausgabe 2/14 der Schweizerischen ZeItschrift FÜR GANZHEITSMEDizin) befinden sich Schlüsselpublikationen zweier Gelehrter, die nicht nur zur gleichen Zeit lebten und kurz hintereinander starben, sondern auch etliche Parallelen in ihren Lebensläufen aufwiesen: «De Historia Stirpium» von Leonhart Fuchs $\left({ }^{\star} 1501\right.$ in Wemding, $\uparrow 1566$ in Tübingen) (Abb. 1) und «Euonymus, sive de Remedijs secretis» sowie "Ander Theil des Schatzs Euonymi von allerhand künstlichen und bewerten Oelen» von Conrad Gessner (* 1516, † 1565 in Zürich) (Abb. 2). Kurz vor ihrem Tod gerieten sie als Konkurrenten bezüglich der geplanten Herausgabe ihrer Pflanzenbücher miteinander in Streit. Ihre Werke konnten sie dann doch nicht mehr vollenden.

\section{Ähnlich und doch anders: Analogien im Werdegang}

Obwohl Fuchs von König Christian III. einen Ruf an die Universität von Kopenhagen erhielt und ihm Herzog Cosimo de Medici den Vorsteherposten im botanischen Garten in Pisa anbot, lehnte dieser insbesondere aus Gründen der protestantischen Religionszugehörigkeit ab. Gessner schlug aus ähnlichen Gründen das Angebot von Johann Jakob Fugger in Augsburg aus, die Erziehung dessen Söhne und Enkel zu übernehmen. Beiden gelang es jedoch, Wappenbriefe von Kaisern zu erhalten: Fuchs im Jahr 1555 von Karl V. und Gessner im April 1564 von Kaiser Ferdinand I.

Dennoch befanden sich die zwei Professoren wegen der hohen Druckkosten für ihre Bücher und der grossen Zahl zu versorgender Familienmitglieder immer wieder in Geldnot und dadurch auch in Abhängigkeit von ihren Gönnern (z.B. Gessner von Zwingli, Myconius sowie Bullinger und Fuchs von Herzog Albrecht von Preussen). Bezüglich der Familienverhältnisse hatte es Fuchs jedoch besser getroffen, als er im Alter von 24 Jahren als Arzt in München die Patriziertochter Anna Friedberg heiratete und mit ihr schliesslich vier Söhne und sechs Töchter grosszog. Gessner verliebte sich mit 19 Jahren in die schöne, aber mittellose Barbara Singeysen, die sich zudem als ständig kränklich und in der Haushaltsführung unfähig erwies. In Zürich durfte er nach der als «ungünstig» beurteilten Heirat daher einige Zeit nur die Stelle eines Elementarlehrers der untersten Klasse bekleiden. Er blieb kinderlos, unterstützte aber ausser seiner alten Mutter noch etliche Neffen und Nichten $[1,2]$.

Auch mit der Pest wurden beide konfrontiert. Fuchs floh allerdings aus Tübingen und wartete einige Jahre in Esslingen das Abflauen der Krankheitswelle ab, während Gessner, der zuvor den erkrankten Bullinger gesundgepflegt hatte, der Seuche erlag.

Beide waren in ihrer Korrespondenz mit Kontrahenten sehr ausführlich und konnten sich an einer Thematik regelrecht festbeissen. Fuchs stritt sich fast 15 Jahre lang mit dem Medizinprofessor Janus Cornarius aus Marburg schriftlich herum. Jener interpretierte Aetius von Amida in den Augen von Fuchs falsch und wurde daher in seiner «Historia stirpium» als unfähig dargestellt. Cornarius konterte mit «Vulpecula excoriata seu refutatio historiae Leonhardi Fuchsii» (Das abgezogene Füchslein, oder Widerlegung der Erzählung des Leonh. Fuchs.) und anderen Schmähschriften, worauf der Angegriffene in Basel 1545 seinen «Cornarius furens» (Rasender Cornarius) veröffentlichte [3].

Gessner konnte ebenfalls äusserst hartnäckig sein. So verwickelte er sich zwischen 1555 und 1565 in einen Streit mit dem als arrogant geltenden italienischen Arzt und Botaniker Pietro Andrea Mattioli. Was mit persönlichen Briefen begann, wurde zunehmend öffentlich publiziert; dabei wurden etliche Freunde und andere Wissenschaftler als «neutrale Gutachter» mit einbezogen. Es ging darum, dass Mattioli ganz offensichtlich einen Eisenhut nach der Beschreibung von Dioskurides zeichnen liess und nicht nach der Natur [4].

\section{KARGER}

Fax +497614520714 Information@Karger.com www.karger.com
Dr. Barbara Brauckmann

Öffentlichkeitsarbeit, Departement Chemie und Angewandte Biowissenschaften ETH Zürich

HCI J 57.2, Vladimir-Prelog-Weg 10, 8093 Zürich, Schweiz

brauckmann@chem.ethz.ch 

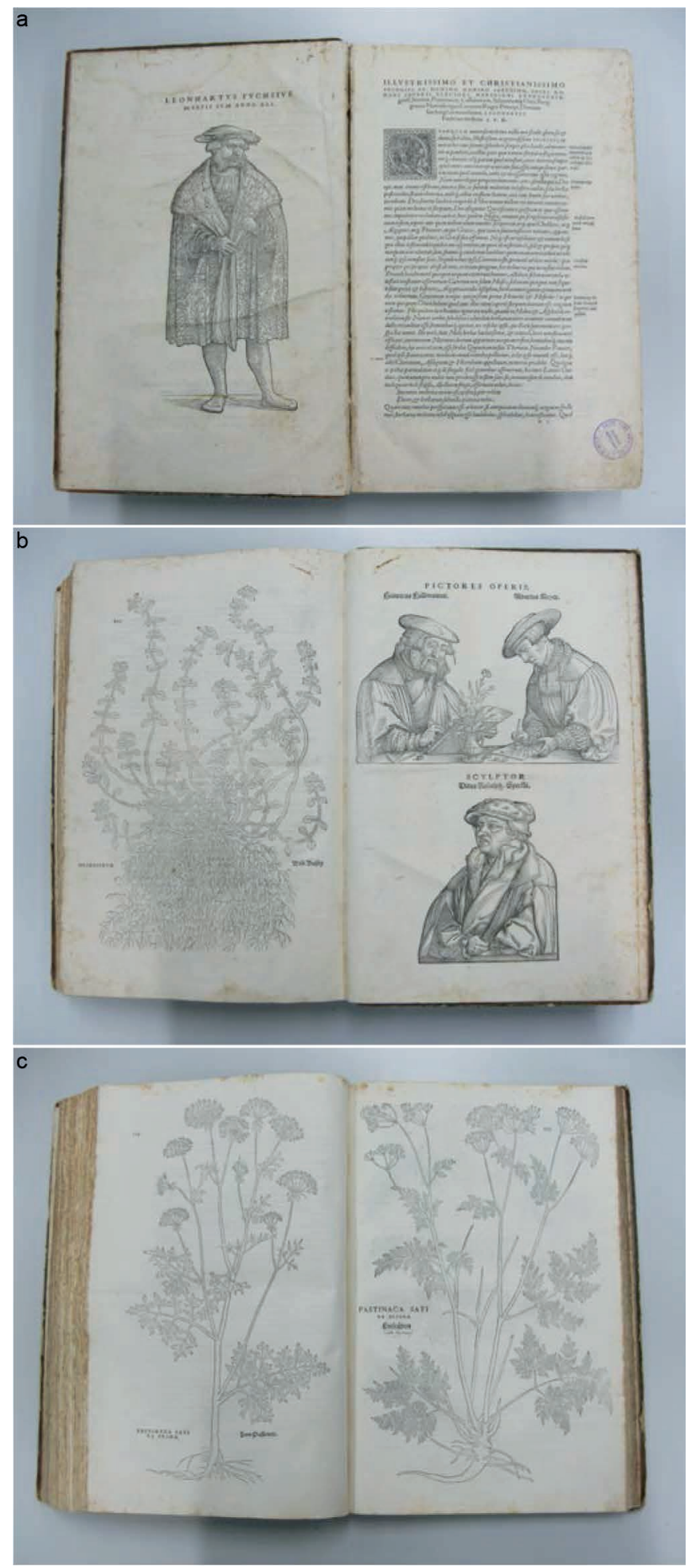

Abb. 1. «De historia stirpium commentarii insignes ...» von Leonhart Fuchs. a Das Titelblatt trägt auf der Rückseite das Bild des Leonhart Fuchs in seinem 41. Lebensjahr. b Das Schlussblatt zeigt die Bildnisse des Strassburger Formschneiders Veyt Rudolff Speckle (um 1505 bis 1550) sowie der Zeichner Heinrich Füllmaurer (um 1500 bis 1547/48) und Albert Meyer (um 1510 bis nach 1561). c Sie malten nach den jeweiligen Anforderungen und Korrekturanweisungen entweder Aquarelle oder übertrugen Vorzeichnungen auf Holzstöcke («Aufreissen»). (Fotos: Departement Chemie und Angewandte Biowissenschaften der ETH Zürich.)

\section{Leonhart Fuchs, der Rektor in Tübingen}

Leonhart Fuchs immatrikulierte sich nach der Lateinschule und einem Studium der Philosophie und «Naturlehre» für die Fächer Griechisch, Lateinisch und Hebräisch. Nach dem Abschluss als «Magister artium» mit Lehrberechtigung nahm er das Medizinstudium auf und promovierte 1524. Später geriet er als Verfechter der Reformationsbewegung in Konflikt mit der konservativen katholischen Universitätsleitung von Ingolstadt, trat dann als Leibarzt in die Dienste des Markgrafen von Brandenburg ein und nahm 1534 schliesslich einen Ruf an die protestantisch zu reformierende Universität Tübingen an.

Dort legte er die Statuten der medizinischen Fakultät fest, lehnte die Astrologie als einen Bereich der Medizin ab, setzte sich für die Schriften der griechischen Ärzte Galen, Hippokrates und Dioskurides ein und gab etwa 1551 in Anlehnung an Vesals gedrucktes Werk von 1543 auch sein Lehrbuch «De corporis humani fabrica» heraus. Zwischen 1535 und 1566 bekleidete er siebenmal das Rektorenamt $[5,6]$.

Fuchs führte in den Sommersemestern botanische Exkursionen in die Umgebung von Tübingen ein. Dort legte er auch einen Garten an, in dem er Pflanzen zu Studienzwecken zog. In seine Vorlesungen integrierte er Pflanzenzeichnungen und Erläuterungen an frischen Pflanzenexemplaren.

Im Jahr 1542 erschien in lateinischer Sprache mit «De Historia Stirpium commmentarii insignes ...»(Kommentare zur Geschichte der Pflanzen) sein erstes Kräuterbuch. Darin wurden über 400 europäische und 100 exotische Pflanzen systematisch unter Berücksichtigung von Standort, Blütezeiten und medizinischen Verwendungsmöglichkeiten («Krafft und Würckung») beschrieben und in 511 Holzschnitten abgebildet. Dabei hatte Fuchs die bereits von den «alten Griechen» aufgeführten Heilpflanzen in der deutschen Flora zu finden versucht. Zudem verglich er das Aussehen von Pflanzen an verschiedenen Fundorten und erkannte Verwandtschaftsbeziehungen zwischen ähnlich aussehenden Pflanzen.

Sein Basler Verleger Michael Isingrin drängte ihn zu einer deutschen Ausgabe für eine über den lateinisch sprechenden Gelehrtenkreis hinausgehende Käuferschicht, die 1543 unter dem Titel «New Kreüterbuch» herausgegeben wurde. So sollten die hohen Kosten für die Anfertigung der Druckstöcke aus Birnbaumholz kompensiert werden. Aber auch weitere verkürzte und verbilligte Fassungen brachten keinen finanziellen «Durchbruch». Manchmal liess Fuchs auch Kopien von Bildern aus anderen Kräuterbüchern und Herbarien, z.B. von Pietro Andrea Mattioli, Rembert Dodoens oder Leonhard Rauwolf, anfertigen [5-7]. 
Insbesondere durch die Konkurrenz mit dem volkstümlicher geschriebenen und auch preisgünstigeren Kräuterbuch des Hieronymus Bock plante er weitere Ausgaben. Bis kurz vor seinem Tod arbeitete er daher beharrlich an seinem grossen Projekt: Es sollte ein dreibändiges Werk mit mehr als 1000 bildhaft dargestellten Heilpflanzen werden.

Als er in jener Zeit im Briefwechsel mit Gessner erfuhr, dass dieser eine ähnliche Arbeit plante, versuchte er ihn von seinem Vorhaben abzuhalten.

\section{Ein aufschlussreicher Briefwechsel}

Der um einige Jahre jüngere Gessner ging jedoch nicht auf die Argumente von Fuchs ein und wollte erst einmal dessen Publikation abwarten. Er schrieb daher:

«... Dein Wunsch geht dahin, dass andere ihre Beobachtungen dir zusenden möchten, es wird mich freuen, wenn dies recht viele Pflanzenliebhaber thun und dadurch dein grosses und schönes Unternehmen unterstützen. Auch ich würde dies gern tun, wenn ich nicht schon so gar vieles gesammelt hätte, oder wenn meine Bemerkungen so ins Reine geschrieben wären, dass sie dir dienen könnten. Aber die Menge meiner Beobachtungen ist auf unzähligen kleinen Blättchen mehr angemerkt als ausgeführt und so geschrieben, dass sie ausser mir selbst niemandem dienen können. ... Ich will warten, bis dein ganzes Werk herausgekommen ist; nach Lesung desselben werde ich dann, wenn ich noch lebe, Zusätze schreiben und sie auch dem Isingrin zum Druck übergeben. ...» [2]

So unterblieb eine Zusammenarbeit, und der Tübinger kommentierte dies am 24 . November 1565 , also wenige Wochen vor Gessners Tod, in einem ungedruckten Brief an seinen Freund und früheren Tübinger Kollegen Joachim Camerarius dem Älteren (1500-1574):

«... Es befremdet mich sehr, dass Gessners angekündigtes Werk bei so vielen so grosse Erwartungen erregt, da er mir noch vor einem Jahr schrieb, er habe noch nicht einmal seine Sammlungen zu Bearbeitung desselben vollständig beieinander. ... Auch wenn er von mir verlangt, dass ich ihm erlaube, meine Pflanzengeschichte nach eignen Absichten zu ordnen, dann wolle er mir Zusätze zu denselben schreiben. Er mag seine eignen Sammlungen ordnen wie er will, aber zu den meinigen räume ich ihm ein solches Recht nicht ein, und werde ihm auch keine Pflanzen mehr senden. ...» [2]

\section{Conrad Gessner, der Universalgelehrte in Zürich}

Gessner hatte wie Fuchs die Klassiker der Antike studiert und übersetzt. Er liess sich nach seiner Promotion zum Doktor der Medizin im Februar 1541 in Zürich nieder, wurde 1546 Professor der Physik, Naturphilosophie und Ethik am Karolinum an der Stiftsschule, 1554 Oberstadtarzt und 1558 zum Chorherrn am Grossmünster ernannt. Auch er gründete einen der ersten Botanischen Gärten sowie eine bedeutende Naturaliensammlung.

Mit 29 Jahren gab er ein Werk zum Druck, in dem das gesamte griechische, lateinische und hebräische Schrifttum vom Altertum bis zu seiner Zeit erfasst war, die «Bibliotheca universalis». Im ersten Teil von 1545
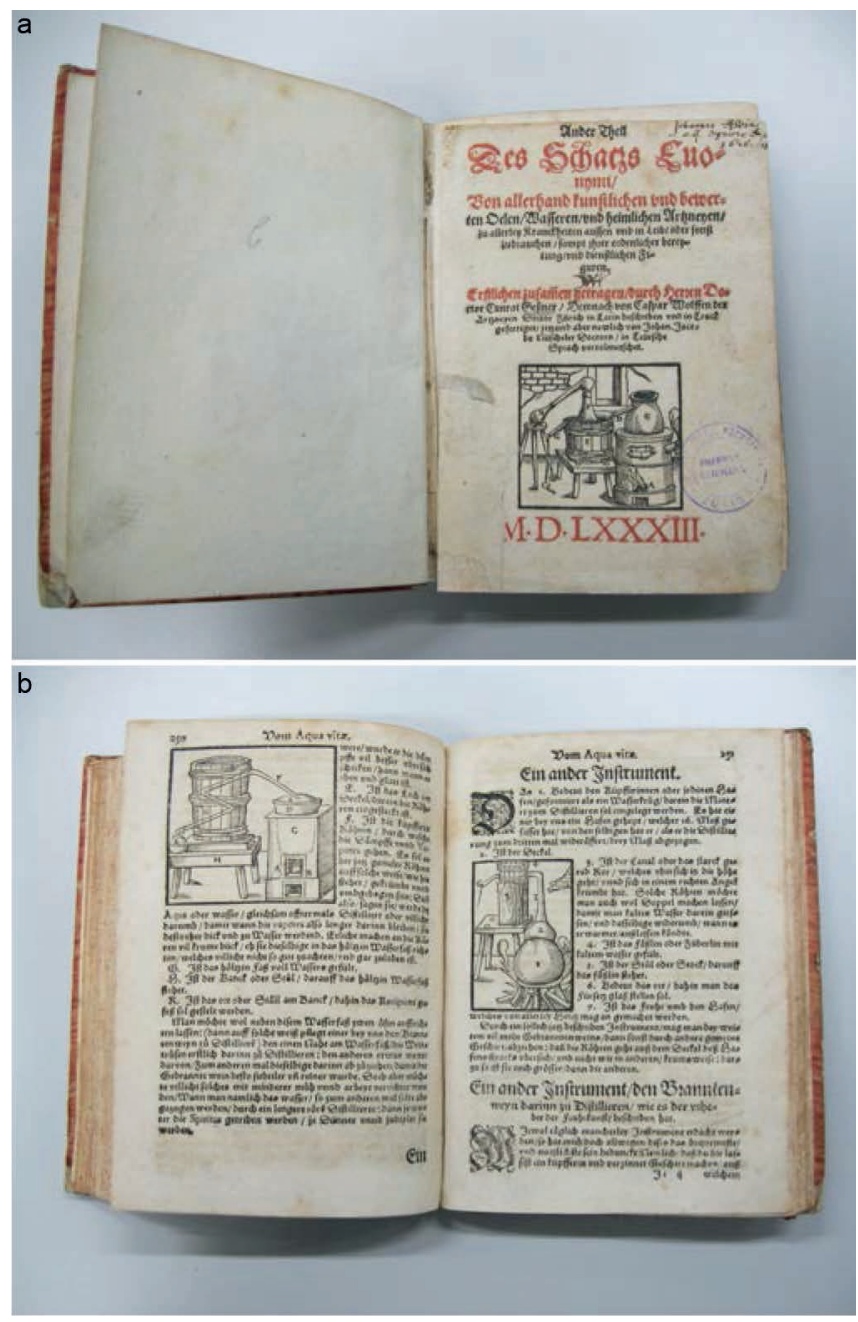

Abb. 2. "Ander Theil des Schatzs Euonymi Von allerhand kunstlichen und bewerten Oelen, Wasseren, und heimlichen Artzneyen zu allerley Kranckheiten aussen und in Leib oder sonst zubrauchen sampt ihrer ordenlicher bereytung und dienstlichen Figuren Erstlichen zusammen getragen durch Herren Doctor Gessner, Demnach von Caspar Wolffen der Artzney Doctor Zürich in Latin beschriben und in Truck gefertiget jetz und aber newlich von Johann Jacobo Nüscheler Doctorn in teütsche Sprach vertolmetschet» von Conrad Gessner. a, b Gessner trug wesentlich zur Darstellung und Verwendung chemischer Arzneien bei, insbesondere durch Destillation zur Herstellung von Wässern und Ölen und zum Ausziehen von Quintessenzen aus mineralischen, pflanzlichen und tierischen Ausgangsmaterialien. Ebenso behandelte er Heizquellen, verschiedenste Ofenformen, Wasserbäder und sonstiges Laboratoriumsinventar [8]. (Fotos: Departement Chemie und Angewandte Biowissenschaften der ETH Zürich.) 
bibliographierte er rund 10000 Werke mit Inhaltsangaben. Der zweite Teilvon 1548 umfasst 19 Bände mit Buchinhalten nach Themen, beginnend mit der Grammatik in Band 1. In dem mehrbändigen Werk der «Historia animalium» sortierte Gessner alles Wissen über die Tiere von der Antike bis ins Mittelalter und fügte neue Kenntnisse und Illustrationen hinzu. In den Jahren 1551 bis 1558 erschienen 4 Foliobände mit insgesamt 4500 Seiten über die Säugetiere, Amphibien und vierfüssigen Reptilien, die Vögel und die Wassertiere, teilweise auch deutsche Übersetzungen. 65 Tafeln legte Gessner selbst an. Postum wurden der 5. Band über die Schlangen (1587) und Band 6 über die Insekten (1634) herausgegeben $[1,2]$.

In «Corpus Venetum de Balneis« (1553) beschrieb er Bädervorschriften und Heilquellen in Deutschland und der Schweiz. In «Thesaurus Euonymi Philiatri de remediis secretis» wurde das damalige Wissen über Destillationskunst, Chemie, Arzneimittel und Medizin zusammengefasst [8].

Am liebsten beschäftigte sich Gessner jedoch mit der Botanik. «Ein kleines Gärtchen, mit seltenen Alpenblumen und ausländischen Pflanzen angefüllt, von ihm selbst gewartet und bebaut, war eine seiner grössten Freuden. Später, als Einkommen und Vermögensumstände sich verbessert hatten, pflanzte er in einem zweiten Garten, was ihm von seinen Freunden aus der Schweiz, Deutschland und Italien und Frankreich Seltenes geschickt wurde.» Um die therapeutischen Eigenschaften von z.B. Helleborus und Tabak beschreiben zu können, testete er diese Pflanzen trotz Vergiftungserscheinungen im Selbstversuch [2].
1561 gab er «De hortis Germaniae» heraus, «in dem einmal in alphabetischer Reihenfolge die Namen jener Pflanzenarten aufgeführt werden, die die Liebhaber der Pflanzengärten in deutschen Landen mit viel Liebe und Eifer in ihrem Garten ziehen, dann aber der meisten Herkunft und Lebensdauer beschrieben wird, dazu was von der Pflege notwendig ist». Im «Catalogus plantarum nomina» stellte er 1542 ein Namenbuch zusammen und erläuterte die Bedeutung von Pflanzenteilen, insbesondere der Blüten und Früchte für die Pflanzensystematik.

Obwohl er selbst gut zeichnete und finanziell unter Druck stand, beauftragte er ähnlich wie Fuchs Zeichner und Holzschneider, die unter seiner Aufsicht die rund 200 Pflanzen nachbilden mussten, die er selbst von Feldern, Wegen, Weinbergen, Gärten, Seen und Bergwiesen mitgebracht hatte. Ein ausgedehntes Korrespondentennetz verband ihn mit Hunderten von Humanisten, mit denen er seine Kenntnisse diskutierte und die ihm etliche Sammlungsobjekte und Pflanzenproben zusandten. So entstand allmählich eine Sammlung von über 1000 gezeichneten und kolorierten Pflanzenabbildungen, aus der Gessner in seinen letzten Lebensjahren eine «Historia Plantarum» (Naturgeschichte der Pflanzenwelt) erstellen wollte und nun seinerseits nicht davon erbaut war, aus Tübingen zu erfahren, dass Fuchs Vergleichbares plante. Als der an der Pest erkrankte Zürcher im Dezember 1565 seinen Nachlass sortiert, finden ihn die Abschied nehmenden Besucher inmitten seines Pflanzenwerkes vor. Es war tatsächlich, wie im Brief an Fuchs erwähnt, erst auf Unmengen kleiner Zettel notiert $[1,2]$.

\section{Literatur}

1 Mähly JA: Gesner, Konrad. http://de.wikisource.org/w/index.php?title= ADB:Gesner,_Konrad_(Naturforscher) ¿oldid $=1706703$.

2 «Aus den Quellen geschöpft von Johannes Hanhart, Stadtpfarrer in Winterthur 1824»: Conrad Gesner - Ein Beytrag zur Geschichte des wissenschaftlichen Strebens und der Glaubensverbesserung im 16ten Jahrhundert.

3 www.ub.unibas.ch/cmsdata/spezialkataloge/ gg/higg0320.html.
4 De Aconito primo Dioscordis herausgeg. von C. Wolf, Zürich 1577. - Delisle C: The Letter: Private Text or Public Place? The Mattioli-Gesner Controversy about the aconitum Primum. Gesnerus 2004;61: 161-176.

5 Baumann-Schleihauf S: Kräuterbücher und die Fuchsie erinnern an Leonhart Fuchs. www.pharmazeutische-zeitung.del indexphp?id=22363.

6 Mayer-Nicolai C: Vergleich der durch die historischen Autoren Hildegard von
Bingen und Leonhart Fuchs pflanzlichen Arzneimitteln zugeschriebenen mit aktuell anerkannten Indikationen.

opus.bibliothek.uni-wuerzburg.de /files/2752/MayerNicolaiDissTeil1.pdf.

7 Rath G: Fuchs, Leonhart. www.deutsche-biographie.del pnd119059487.html.

8 Dobler F: Conrad Gessner als Pharmazeut. Promotionsarbeit ETH Zürich, 1955. http://e-collection.library.ethz.ch/eserv/ eth:34390/eth-34390-02.pdf. 